\title{
SIMULAÇÃO E ANÁlISE DA HIDRATAÇÃO DE SOJA TRANSGẾNICA E CONVENCIONAL
}

\author{
D. J. NICOLIN ${ }^{1}$, A. C. SANTOS ${ }^{2}$, G. L. GONÇALVES ${ }^{1}$, R. M. M. JORGE ${ }^{2}$, L. M. M. \\ JORGE $^{1}$ \\ ${ }^{1}$ Universidade Estadual de Maringá, Departamento de Engenharia Química, Programa de Pós- \\ graduação em Engenharia Química \\ ${ }^{2}$ Universidade Federal do Paraná, Departamento de Engenharia Química \\ E-mail para contato: douglas.nicolin@gmail.com/lmmj@deq.uem.br
}

\begin{abstract}
As características de hidratação da soja convencional (cultivar CD 202) e transgênica (cultivar CD 202 RR) a várias temperaturas foram analisadas a partir do ajuste de um modelo de parâmetros concentrados com variações do volume dos grãos ao longo do processo. $\mathrm{O}$ modelo foi obtido a partir de um balanço de massa eé representado por uma equação diferencial que considera a variação do volume proporcional à massa de água absorvida. A solução do modelo e o ajuste de seus parâmetros foram feitos utilizando-se rotinas compiladas no software MATLAB. Os resultados revelam que o modelo e a técnica de otimização dos parâmetros foram adequados, possibilitando concluir que, na faixa de temperaturas operacionais explorada, a hidratação da soja convencional e da soja transgênica pode ser bem representada. Adicionalmente foram avaliadas as influências da temperatura e da transgenia sobre os parâmetros ajustados, sobre a umidade de equilíbrio e sobre os perfis de umidade do grão ao longo do tempo.
\end{abstract}

\section{INTRODUÇÃO}

A importância da hidratação de grãos de soja reside no fato de que a estrutura e textura dos grãos devem ser modificadas com intuito de reduzir a energia requerida para a moagem dos grãos e aumentar a extração de proteínas e emulsificação de gorduras. Isto é feito pelo amaciamento da estrutura celular dos grãos por meio da hidratação (WANG et al., 1979). A extração da proteína presentes nos grãos é um processo fundamental na indústria de alimentos à base de soja e o consumo de tais alimentos está associado à redução de doenças crônicas não-infecciosas como doenças cardiovasculares, alguns tipos de câncer e osteoporose, pois a soja é rica em isoflavonas(ESTEVES; MONTEIRO, 2001; SILVA et al., 2006).

Para o processamento e obtenção de proteína de soja são usados, em sua maioria, grãos provenientes de cultivares convencionais. Cultivares de soja transgênicos são obtidos por meio da inserção de um gene, na planta, que é resistente ao herbicida glifosato com o intuito de aumentar a resistências dessas plantas a ervas daninhas e reduzir o custo de produção (PELAEZ; ALBERGONI; GUERRA, 2004). Embora a soja transgênica apresente estas vantagens, vários países apresentam resistência ao consumo de alimentos à base de soja transgênica (LUNA et al., 2013). Como o crescimento da produção de soja transgênica no Brasil vem aumentando (ROESSING; LAZZAROTTO, 2006), se torna interessante analisar como ocorre a cinética de hidratação deste tipo de grão.

De maneira geral a hidratação é feita estipulando-se o tempo de imersão empiricamente sendo o tempo de imersão frequentemente utilizado o de 12h (CIABOTTI et al., 2009). Como há interesses em redução de energia de moagem dos grãos, uma descrição precisa da cinética 
da hidratação é de suma importância. Para tal tarefa utilizam-se modelos matemáticos que são validados com dados experimentais de umidade em função do tempo. Modelos fenomenológicos de parâmetros concentrados apresentam base física rigorosa, pois são baseados em etapas elementares de transferência de massa. A variável umidade, nestes modelos, é considerada uniformemente distribuída no interior dos grãos. Tais modelos já foram utilizados com sucesso na descrição da hidratação de grãos de soja convencional (COUTINHO et al., 2005, 2007).

Neste contexto o objetivo do presente trabalho foi modelar matematicamente a cinética da hidratação de soja convencional e transgênica por meio de um modelo fenomenológico de parâmetros concentrados com coeficiente de transferência de massa e volume variáveis. $\mathrm{O}$ modelo foi validado por dados experimentais de hidratação de soja convencional e transgênica. Os parâmetros do modelo foram obtidos por ajuste utilizando-se o método dos mínimos quadrados. A influência da temperatura foi analisada no comportamento do modelo e de seus parâmetros para ambos os cultivares e foi também discutida a influência que a transgenia causa na cinética de hidratação de grãos de soja.

\section{TEORIA}

O modelo fenomenológico de parâmetros concentrados utilizado para modelar a hidratação de soja convencional e transgênica foi proposto inicialmente por Coutinho et al. (2007). Este modelo foi obtido por meio de um balanço de massa global no grão de soja considerado esférico e este balanço de massa estabelece que o acúmulo de massa no interior dos grãos ocorre devido à convecção natural. O fluxo convectivo de água é dado por $N_{A}=K_{S}\left(\rho_{e q}-\rho_{A}\right)$. A Equação 1 apresenta o balanço de massa. A Equação 2 apresenta a forma do balanço de massa quando se considera que o volume dos grão varia ao longo da hidratação.

$$
\begin{aligned}
& \frac{d\left(\rho_{A} V\right)}{d t}=K_{S} A\left(\rho_{e q}-\rho_{A}\right) \\
& V \frac{d \rho_{A}}{d t}+\rho_{A} \frac{d V}{d t}=K_{S} A\left(\rho_{e q}-\rho_{A}\right)
\end{aligned}
$$

Em seu trabalho Coutinho et al. (2007) consideraram que a variação do volume dos grãos ao longo da hidratação é diretamente proporcional ao acúmulo de massa no sistema, ou seja, $\Delta V=\alpha \Delta m$. Neste trabalho os autores demonstraram experimentalmente que o valor da constante de proporcionalidade entre variação de volume e de massa é constante e vale $\alpha=1 \mathrm{~cm}^{3} / \mathrm{g}$. Com as manipulações algébricas adequadas é possível escrever a relação entre volume e massa como é apresentado pela Equação 3.

$$
V=V_{0} \frac{\left(1-\alpha \rho_{A 0}\right)}{\left(1-\alpha \rho_{A}\right)}
$$

Para completar o modelo calcula-se a derivada da Equação 3 em função do tempo e o resultado é substituído na Equação 2. O resultado $\alpha=1 \mathrm{~cm}^{3} / \mathrm{g}$ também é substituído na Equação 2. Outra alteração que é feita no modelo é a inserção da dependência do coeficiente de transferência de massa $\left(K_{S}\right)$ com a umidade $\left(\rho_{A}\right)$. Em seu trabalho Coutinho et al. (2007) consideraram que o coeficiente de transferência de massa varia exponencialmente com a umidade por meio da equação $K_{S}=B_{1} e^{B_{2} \rho_{A}} \mathrm{O}$ modelo final é apresentado pela Equação 4 e é 
composto pela equação diferencial em função do tempo e pela condição inicial necessária para a solução do modelo.

$\frac{d \rho_{A}}{d t}=\frac{6 B_{1} e^{B_{2} \rho_{A}}}{C}\left(\rho_{e q}-\rho_{A}\right)\left(1-\rho_{A}\right)^{4 / 3}$

$\rho_{A}(0)=\rho_{A 0}$

sendo $C=\left[\frac{6 V_{0}}{\pi}\left(1-\rho_{A 0}\right)\right]^{1 / 3}$ e $B_{1}$ e $B_{2}$ os parâmetros do modelo a serem ajustados.

\section{MATERIAIS E MÉTODOS}

Os cultivares de soja utilizados foram o CD 202 (convencional) e o CD 202 RR (transgênico). Ambos são do mesmo cultivar sendo que o termo RR (RoundupReady) indica a variedade transgênica do cultivar CD 202. Os cultivares foram fornecidos pela Cooperativa Agropecuária MourãoenseLtda (COAMO).

Para a obtenção dos valores deu umidade em função do tempo, preparou-se inicialmente o banho termostático (Marconi MA184) com água destilada, que foi mantido nas temperaturas de $10,20,30,40$ e $50^{\circ} \mathrm{C}$ para cada teste experimental de cada cultivar.Para cada teste, 3 litros de uma solução de benzoato de sódio $0,2 \%(\mathrm{~m} / \mathrm{m})$ foram preparados e adicionados num recipiente dividido em duas partes (1,5 litros em cada parte), cada uma das partes foi utilizada para a hidratação de um cultivar de soja. Logo, a hidratação do cultivar convencional e do transgênico ocorreram simultaneamente para cada temperatura. $\mathrm{O}$ benzoato de sódio foi utilizado como agente conservante na água de hidratação com o objetivo de inibir a proliferação de microrganismos durante os longos tempos de imersão dos grãos em água. Aguardou-se o tempo necessário para que a água de hidratação atingisse a temperatura estabelecida. Assim que isto ocorreu, adicionou-se $300 \mathrm{~g}$ de cada cultivar em suas respectivas divisões, devidamente identificadas, do recipiente (este que já estava em contato com o banho termostático e na temperatura ideal para o teste). Amostras foram retiradas em tempos préestabelecidos depois que a contagem do tempo foi iniciada. O excesso de umidade foi retirado com o uso de papel toalha $\mathrm{e}$ as amostras foram adicionadas em pequenos recipientes devidamente identificados. A massa das amostras foi medida e as mesmas foram inseridas em estufa (Fanem $315 \mathrm{SE}$ ) durante $24 \mathrm{~h}$ a $105^{\circ} \mathrm{C}$ para obtenção da umidade de cada amostra (LUTZ, 1985).

Com o objetivo de resolver o modelo para ambos os cultivares e ajustar os parâmetros ótimos para a hidratação de cada cultivar desenvolveram-se rotinas no software MATLAB. Tais rotinas resolveram o modelo utilizando-se a rotina "ode45", que é baseada da fórmula de Runge-Kutta (4,5) (DORMAND; PRINCE, 1980). Os parâmetros do modelo foram ajustados pelo método dos mínimos quadrados por meio da minimização de uma função-objetivo quadrática (Equação 5). O método de otimização dos parâmetros usado foi o método de Levenberg-Marquardt(LEVENBERG, 1944; MARQUARDT, 1963) que está presente no MATLAB no comando "nlinfit".

$$
\phi=\sum_{i=1}^{N}\left(\rho_{\text {Acalc }}^{i}-\rho_{\text {Aexp }}^{i}\right)^{2}
$$


O comando "nlpredci" foi utilizado em conjunto com o comando "nlinfit" para a obtenção dos intervalos de predição dos dados experimentais com 95\% de confiança.

\section{RESULTADOS E DISCUSSÃO}

A Figura 1 apresenta os dados experimentais de hidratação em função do tempo para os cultivares CD 202 e CD $202 \mathrm{RR}$ a $10^{\circ} \mathrm{C}$. Além disso esta figura ainda apresenta para cada caso o intervalo de predição dos dados calculados com $95 \%$ de confiança. É possível observar que a variabilidade dos dados foi maior nas medições feitas para o cultivar CD 202 RR, embora elas apresentem uma proporção muito similar. Outro fato pode ser observado é que há superposição dos intervalos de predição, o que confirma que os dados para ambos os cultivares não são significativamente diferentes (JEAN-PIERRE; JEAN-PIERRE; COROLLER, 2010). Isto sugere que a transgenia que difere os cultivares em questão não interferiu nos dados de hidratação. Esta tendência se manteve para as demais temperaturas consideradas.
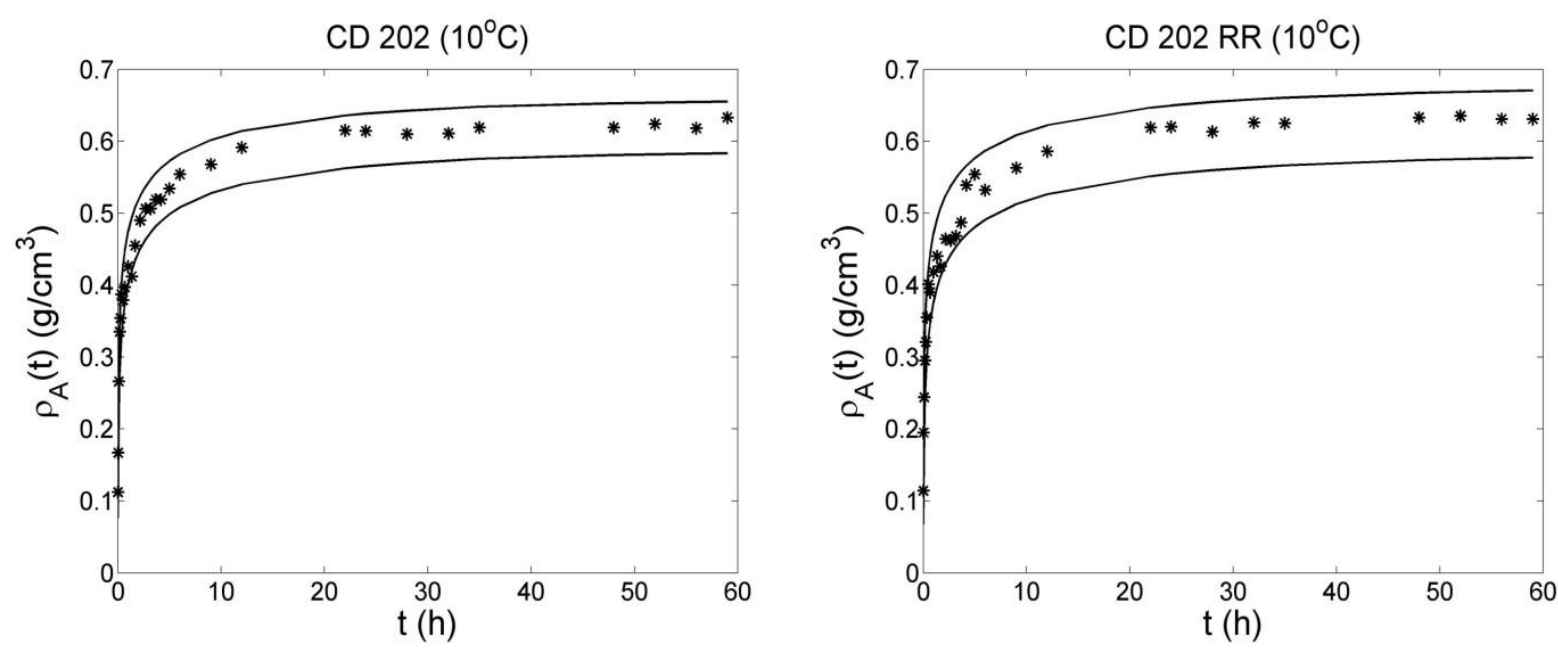

Figura 1: Umidades experimentais em função do tempo e intervalos de predição com 95\% de confiança.

Os modelos apresentaram baixos valores do resíduo quadrático ao fim das iterações da rotina de ajuste dos parâmetros e solução dos modelos, indicando um bom ajuste do modelo para ambos os cultivares (Tabela 1). Embora haja pequenas diferenças nos valores dos resíduos, para ambos os cultivares e em todas as temperaturas consideradas a ordem de grandeza do desvio entre as previsões dos modelos e os dados experimentais teve a mesma ordem de grandeza.

Tabela 1: Resíduos quadráticos dos ajustes.

\begin{tabular}{|c|c|c|}
\hline $\mathrm{T}\left({ }^{\circ} \mathrm{C}\right)$ & \multicolumn{2}{|c|}{$\phi \times 10^{3}\left(\mathrm{~g}^{2} / \mathrm{m}^{6}\right)$} \\
\hline & $\mathrm{CD} 202$ & $\mathrm{CD} 202 \mathrm{RR}$ \\
\hline 10 & 5,51 & 9,25 \\
\hline 20 & 6,67 & 11,8 \\
\hline 30 & 4,06 & 5,63 \\
\hline 40 & 10,4 & 6,38 \\
\hline 50 & 4,35 & 2,56 \\
\hline
\end{tabular}


Os parâmetros do modelo para ambos os cultivares são apresentados em função da temperatura nas Figuras $2\left(B_{1}\right)$ e Figura $3\left(B_{2}\right)$. O parâmetro $B_{1}$ apresentou valores na mesma ordem de grandeza até a temperaturas de $40^{\circ} \mathrm{C}$ para ambos os cultivares, porém o valor deste parâmetro foi maior para o cultivar $\mathrm{CD} 202$ na temperatura de $50^{\circ} \mathrm{C}$. O parâmetro $B_{2}$ apresentou valores na mesma faixa para ambos os cultivares em todas as temperaturas consideradas. Os valores obtidos para este parâmetro foram negativos para ambos os cultivares. Este fato já era esperado uma vez que $B_{2}$ é o fator exponencial do coeficiente de transferência de massa $\left(K_{S}=B_{1} e^{B_{2} \rho_{A}}\right)$ e valores negativos para o fator exponencial faz com que $K_{S}$ tenha um comportamento de decaimento em função de $\rho_{A}$. Este comportamento tem coerência física, pois quanto mais próximo do valor de equilíbrio $\left(\rho_{A} \rightarrow \rho_{e q}\right)$, menor é a transferência de massa e valores menores de $K_{S}$ são esperados.
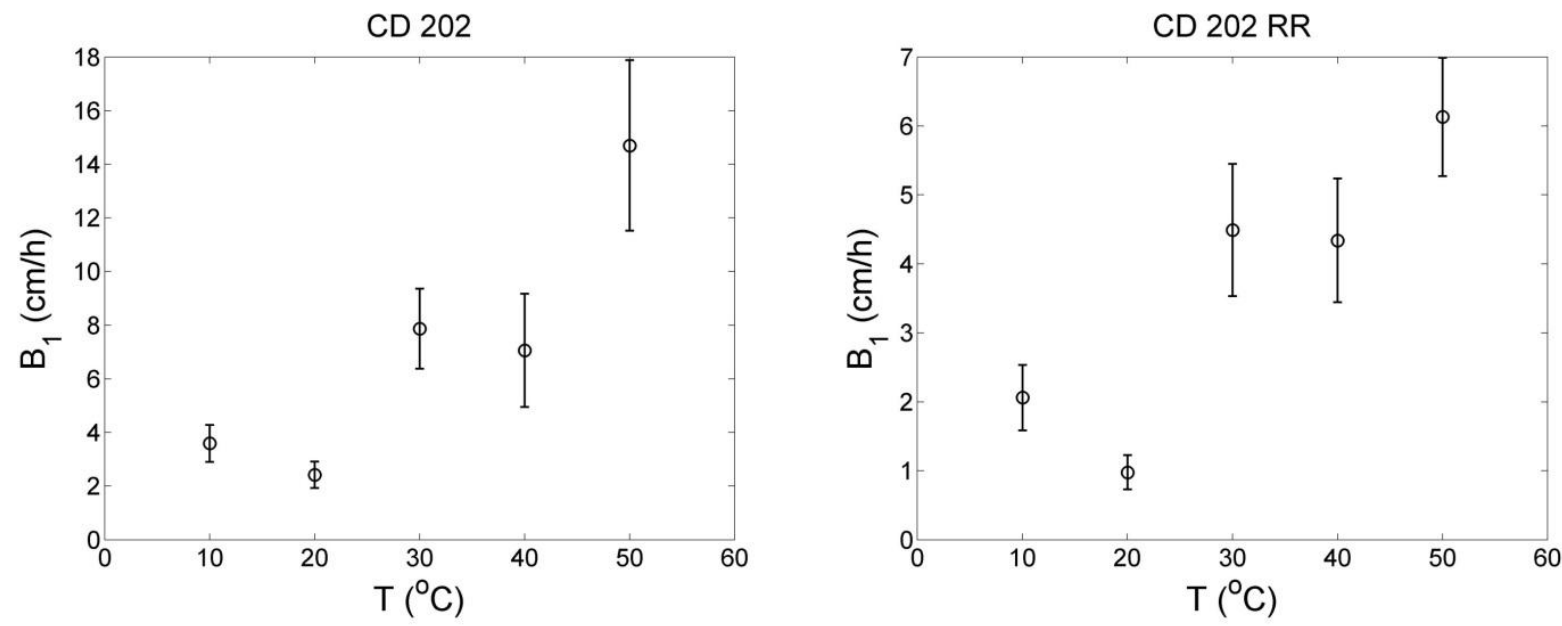

Figura 2: $B_{1}$ em função da temperatura.
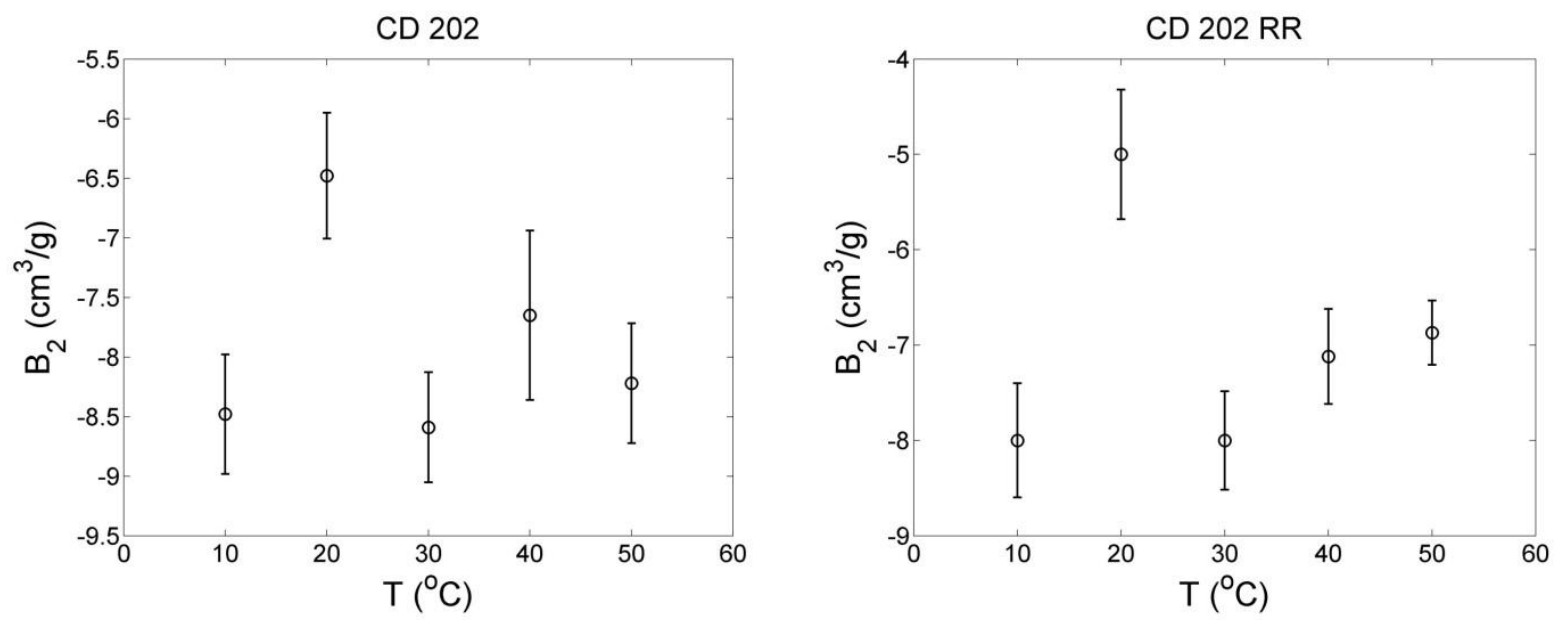

Figura 3: $B_{2}$ em função da temperatura.

As principais tendências dos dados experimentais foram representadas pelo modelo proposto para os cultivares convencional e transgênico como pode ser visto na Figura 4. Esta figura apresenta os perfis de umidade para as temperaturas de 10,30 e $50^{\circ} \mathrm{C}$ num tempo de hidratação mais reduzido para que a visualização dos resultados fosse facilitada, porém um 
estado de equilíbrio já se inicia para o tempo de hidratação mostrado na figura. A umidade de equilíbrio aumenta levemente com a temperatura. Para tempos mais longos as umidades previstas para um estado mais próximo ao de equilíbrio diferem cada vez menos e a influência da temperatura não é mais tão pronunciada.
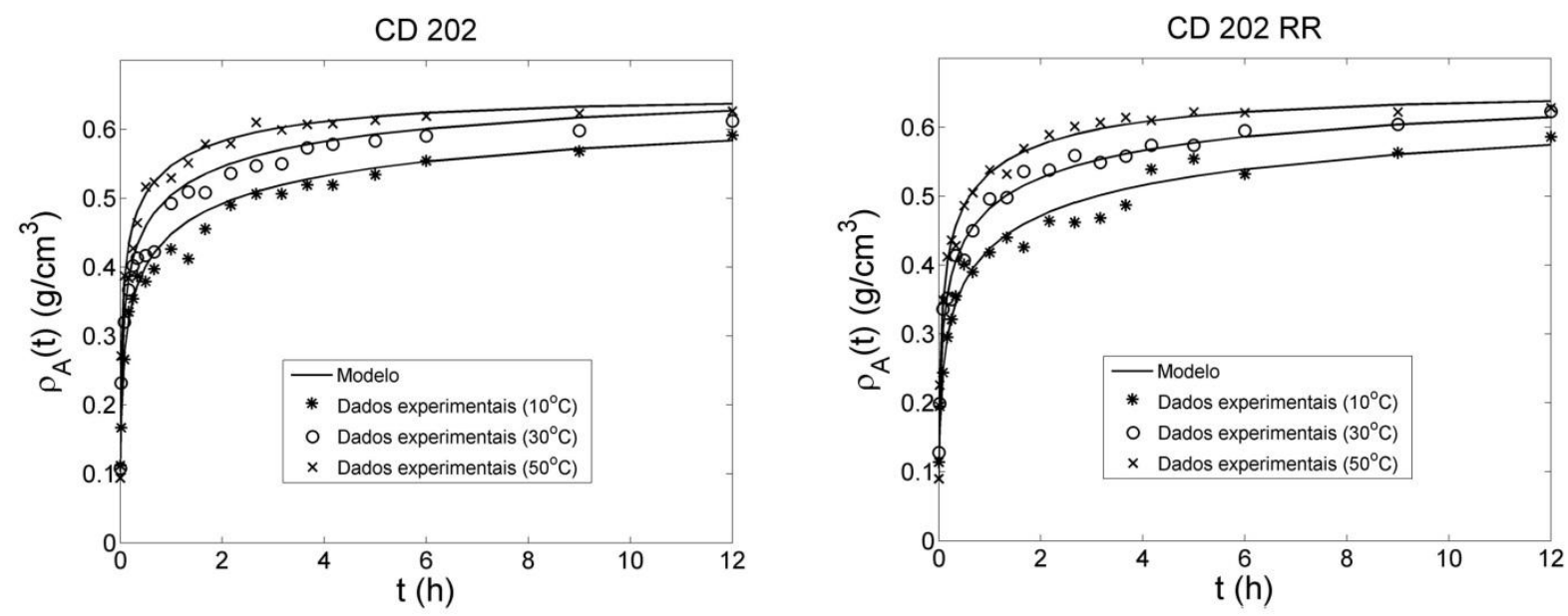

Figura 4: Perfis de umidade em função do tempo para várias temperaturas.

A Figura 5 apresenta o comportamento do coeficiente de transferência $\left(K_{S}\right)$ em função da umidade $\left(\rho_{A}\right)$. Como esperado $K_{S}$ diminuiu exponencialmente com o aumento de $\rho_{A}$, atingindo valores muito próximos de zero conforme $\rho_{A}$ tendia ao valor de equilíbrio $\left(\rho_{e q}\right)$. Este comportamento de $K_{S}$ representa a diminuição gradativa da transferência de massa conforme um estado de equilíbrio vai sendo alcançado. Outro fato que é perceptível na Figura 5 é que o cultivar transgênico (CD 202 RR) apresentou valores para o coeficiente de transferência de massa menores do que o cultivar convencional (CD 202). Isto sugere que a entrada de água nos grãosdo cultivar transgênico sofreu uma maior resistência à transferência de massa que pode ser atribuída a uma estrutura interna mais rígida dos próprios grãos deste cultivar, sugerindo que há características texturais diferentes entre os dois cultivares estudados.
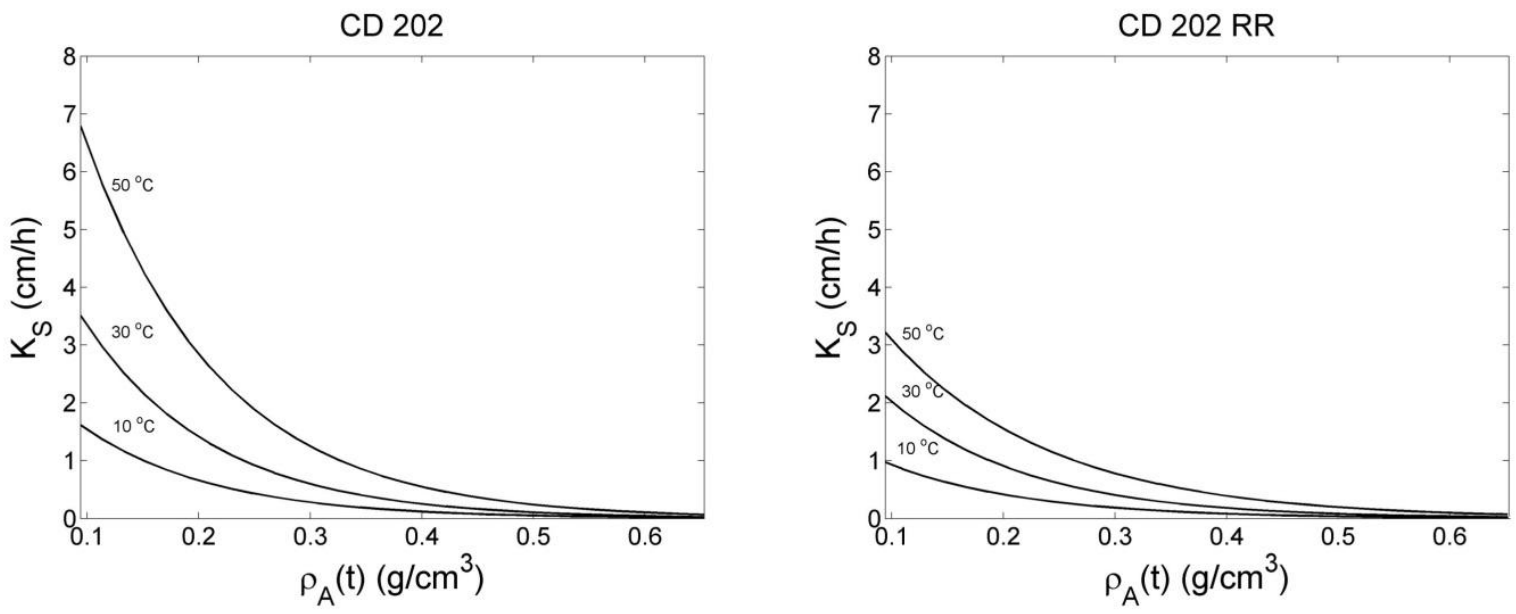

Figura 5: $K_{S}$ em função de $\rho_{A}$. 


\section{CONCLUSÕES}

A transgenia existente entre os cultivares CD 202 e CD 202 RR não afetou o comportamento da curva de hidratação e isto pode ser concluído pela superposição existente entre os intervalos de predição dos dados com $95 \%$ de confiança.

O modelo proposto representou satisfatoriamente as principais tendências dos dados experimentais em todas as temperaturas consideradas, mesmo havendo algumas diferenças nos valores dos parâmetros obtidos para todos os casos.

Embora a transgenia não tenha afetado significativamente a cinética de hidratação dos cultivares, a absorção de água ocorreu com maior velocidade para o cultivar convencional, uma vez que para este cultivar os valores do coeficiente de transferência de massa foram maiores. A entrada de água nos grãos transgênicos sofreu uma resistência maior sugerindo que os grãos deste cultivar possuem uma estrutura mais rígida e características texturais que desaceleram a absorção de água em comparação com o cultivar convencional.

Devido à qualidade dos ajustes do modelo proposto ter sido satisfatória, este modelo pode ser utilizado na obtenção de curvas de cinética de hidratação de grãos bem como no projeto de unidades de hidratação, estas que dependem do conhecimento da cinética deste processo.

\section{NOMENCLATURA}
A Área superficial dos grãos $\left(\mathrm{cm}^{2}\right)$
$B_{1} \quad$ Fator pré-exponencial $(\mathrm{cm} / \mathrm{h})$
$B_{2} \quad$ Fator exponencial $\left(\mathrm{cm}^{3} / \mathrm{g}\right)$
$i \quad$ Índice de somatório
$K_{S} \quad$ Coeficiente de transferência de massa $(\mathrm{cm} / \mathrm{h})$
$N \quad$ Número máximo de valores no somatório
$N_{A} \quad$ Fluxo de água $\left(\mathrm{g} / \mathrm{cm}^{2} . \mathrm{h}\right)$
$t \quad$ Tempo (h)
$V \quad$ Volume dos grãos $\left(\mathrm{cm}^{3}\right)$
$V_{0} \quad$ Volume inicial dos grãos $\left(\mathrm{cm}^{3}\right)$

\section{$\underline{\text { Símbolos gregos }}$}

$\alpha \quad$ Coeficiente de proporcionalidade $\left(\mathrm{cm}^{3} / \mathrm{g}\right)$

$\rho_{A} \quad$ Concentração de água nos grãos $\left(\mathrm{g} / \mathrm{cm}^{3}\right)$

$\rho_{A 0}$ Concentração inicial de água nos grãos $\left(\mathrm{g} / \mathrm{cm}^{3}\right)$

$\rho_{\text {Acalc }}^{i}$ Concentração de água calculada pelo modelo $\left(\mathrm{g} / \mathrm{cm}^{3}\right)$

$\rho_{\text {Aexp }}^{i}$ Concentração de água experimentais $\left(\mathrm{g} / \mathrm{cm}^{3}\right)$

$\rho_{e q}$ Concentração de água de equilíbrio $\left(\mathrm{g} / \mathrm{cm}^{3}\right)$

$\phi \quad$ Função objetivo $\left(\mathrm{g}^{2} / \mathrm{cm}^{6}\right)$

\section{REFERÊNCIAS BIBLIOGRÁFICAS}

CIABOTTI, S.; BARCELOS, M. F. P.; CIRILlO, M. A.; PINHEIRO, A. C. M. Propriedades tecnológicas e sensoriais de produto similar ao tofu obtido pela adição de soro de leite 
ao extrato de soja Sensorial and technologic properties of product similar to tofu obtained with whey and soymilk addiction. Ciencia e Tecnologia de Alimentos, v. 29, n. 2, p. 346-353, 2009.

COUTINHO, M. R.; OMOTO, E. S.; ANDRADE, C. M. G.; JORGE, L. M. M. Modelagem e validação da hidratação de grãos de soja. Ciência e Tecnologia de Alimentos, v. 25, n. 3, p. 603-610, set. 2005. Disponível em: <http://www.scielo.br/scielo.php?script=sci_arttext\&pid=S0101$20612005000300034 \& \operatorname{lng}=\mathrm{pt} \& n \mathrm{~m}=\mathrm{iso} \& \mathrm{t} \operatorname{lng}=\mathrm{pt}>$. Acesso em: 2 dez. 2013.

COUTINHO, M. R.; CONCEIÇÃO, W. A. S.; OMOTO, E. S.; ANDRADE, C. M. G.; JORGE, L. M. M. Novo modelo de parâmetros concentrados aplicado à hidratação de grãos. Ciência e Tecnologia de Alimentos, v. 27, n. 3, p. 451-455, set. 2007. Disponível em: $\quad<$ http://www.scielo.br/scielo.php?script=sci_arttext\&pid=S0101$20612007000300005 \& \operatorname{lng}=$ pt\&nrm=iso\&tlng=pt $>$. Acesso em: 2 dez. 2013.

DORMAND, J. R.; PRINCE, P. J. A family of embedded Runge-Kutta formulae. Journal of Computational and Applied Mathematics, v. 6, n. 1, p. 19-26, mar. 1980. Disponível em: <http://linkinghub.elsevier.com/retrieve/pii/0771050X80900133>. Acesso em: 17 jan. 2014.

ESTEVES, E. A.; MONTEIRO, J. B. R. Efeitos Benéficos das Isoflavonas de Soja em Doenças Crônicas. Rev. Nutr., v. 14, n. 1, p. 43-52, 2001.

JEAN-PIERRE, G.; JEAN-PIERRE, V.; COROLLER, L. New Prediction Interval and Band in the Nonlinear Regression Model: Application to Predictive Modeling in Foods. Communications in Statistics - Simulation and Computation, v. 39, n. 2, p. 322-334, fev. $2010 . \quad$ Disponível em: <http://www.tandfonline.com/doi/abs/10.1080/03610910903448799>. Acesso em: 26 mar. 2014.

LEVENBERG, K. A method for the solution of certain problems in least squares. Quart. Applied Math., v. 2, p. 164-168, 1944.

LUNA, A. S.; PINHO, J. S. A.; FERRÉ, J.; BOQUÉ, R.Rapid characterization of transgenic and non-transgenic soybean oils by chemometric methods using NIR spectroscopy. Spectrochimica acta. Part A, Molecular and biomolecular spectroscopy, v. 100, p. 1159, 1 jan. 2013. Disponível em: <http://www.ncbi.nlm.nih.gov/pubmed/22502875>. Acesso em: 14 mar. 2014.

LUTZ, I. A. Normas analíticas do Instituto Adolfo Lutz. São Paulo: [s.n.], 1985.

MARQUARDT, D. W. An Algorithm for Least-Squares Estimation of Nonlinear Parameters. Journal of the Society for Industrial and Applied Mathematics, v. 11, n. 2, p. 431-441, jun. 1963. Disponível em: <http://epubs.siam.org/doi/abs/10.1137/0111030>. Acesso em: 19 nov. 2013.

PELAEZ, V.; ALBERGONI, L.; GUERRA, M. P. Soja Transgênica versus Soja Convencional: Uma Análise Comparativade Custose Benefícios. Cadernos de Ciência e Tecnologia, v. 21, n. 2, p. 279-309, 2004.

ROESSING, A. C.; LAZZAROTTO, J. J. Soja Transgênica no Brasil: Situação Atual e Perspectivas para os Próximos Anos. Reunião de Pesquisa de Soja da Região Central do Brasil, p. 31 - 32, 2006.

SILVA, M. S.; NAVES, M. M.; OLIVEIRA, R. B.; LEITE, O. S. M. Composição Química e Valor Protéico do Resíduo de Soja em Relação ao Grão de Soja 1. Ciencia e Tecnologia de Alimentos, v. 26, n. 3, p. 571-576, 2006.

WANG, H. L.; SWAIN, E. W.; HESSELTINE, C. W.; HEATH, H. D.Hydration of Whole Soybeans Affects Solids Losses and Cooking Quality. Journal of Food Science, v. 44, n. 5, p. 1510-1513, set. 1979. Disponível em: <http://doi.wiley.com/10.1111/j.13652621.1979.tb06474.x>. Acesso em: 27 nov. 2013. 